\title{
INFECTIOUS DISEASE CONTROL POLICY: A ROLE FOR SIMULATION
}

\author{
Margaret L. Brandeau \\ Dept. of Management Science and Engineering \\ Stanford University \\ Stanford, CA 94305 USA
}

\begin{abstract}
Control of infectious diseases is a key global health priority. This paper describes the role that simulation can play in evaluating policies for infectious disease control. We describe ongoing simulation studies in three different areas: HIV prevention and treatment, contact tracing, and hepatitis B prevention and control.
\end{abstract}

\section{INTRODUCTION}

Infectious diseases cause approximately $25 \%$ of deaths worldwide, and $50 \%$ of premature deaths (World Health Organization 1999). The leading infectious disease killers are acute respiratory infections (such as pneumonia and influenza), which account for 3.5 million deaths annually, HIV/AIDS (2.9 million deaths annually), diarrheal diseases (2.2 million deaths annually), tuberculosis (1.6 million deaths annually), malaria (1.1 million deaths annually), and measles (1 million deaths annually) (World Health Organization 1999; World Health Organization 2000). The majority of deaths from communicable disease are preventable: interventions such as vaccination or prevention programs can be given to uninfected people, and appropriate treatment such as antibiotics or antiviral medication can be given to infected people. Infectious diseases can also cause a significant diminution in quality of life: often, people infected with such a disease may live for many years but in a state of poor health. Because of the significant morbidity and mortality caused by infectious disease, control of such diseases is a key global health priority.

Unfortunately, resources for controlling infectious diseases fall far short of estimated need. Thus, it is critical that available disease control funds be used in the most effective manner. To make the best possible resource allocation decisions, policy makers need to know not only the likely effectiveness of alternative disease control programs, but also the likely cost-effectiveness of the programs; this is the level of health benefit achieved for a given investment in disease control (Gold, Siegel et al. 1996).

Evaluating the likely effect of alternative disease control programs is complicated by the fact that infectious diseases grow nonlinearly: the rate at which an infectious disease spreads is, broadly speaking, a function of the number of uninfected people multiplied by the number of infected people (Anderson and May 1991). In order to know how many infections may be averted (or how many life years may be gained) by any given control policy, numerical simulation is needed. Moreover, because of the many uncertainties surrounding the transmission and progression of infectious diseases - such as behavioral and biological factors associated with transmission risk and response to treatment - stochastic simulation is a useful tool for evaluating the likely impact of alternative control programs.

This paper describes the role that simulation can play in evaluating policies for infectious disease control. We describe ongoing simulation studies in three different areas: HIV prevention and treatment, contact tracing, and hepatitis B prevention and control.

\section{HIV PREVENTION AND TREATMENT}

Worldwide, some 33 million people are currently living with HIV/AIDS, with another 2.5 million becoming newly infected each year (World Health Organization 2007). The epidemic varies significantly in different regions of the globe. For example, in sub-Saharan Africa, HIV is spread primarily through heterosexual and mother-tochild transmission, whereas in Eastern Europe and Russia, HIV is spread primarily via injection drug use. In the United States, homosexual contact is a key mode of HIV transmission. Resources for HIV prevention and treatment also vary markedly in different regions of the world. In the United States and other developed countries, a high fraction of patients who are eligible for HIV treatment receive the drugs, whereas in poorer regions such as subSaharan Africa, few eligible people receive HIV treatment. Because of these variations in the epidemic and in 


\section{Brandeau}

available resources to combat the epidemic, programs that are cost-effective in one country may not be cost-effective elsewhere.

\subsection{HIV Treatment in Russia}

Russia is experiencing one of the fastest growing HIV epidemics in the world, with approximately $90 \%$ of cases occurring after 2000 (AIDS Foundation East West 2005; World Health Organization (WHO) 2005). The epidemic has been fueled largely by injection drug use (Joint United Nations Programme on HIV/AIDS (UNAIDS) 2004; Rhodes, Sarang et al. 2004; World Health Organization (WHO) 2005). Currently, few eligible HIV-infected patients in Russia receive HIV treatment, and almost no injection drug users receive treatment. Such therapy, known as highly active antiretroviral therapy (HAART) has two benefits: it significantly extends life of infected individuals, thus providing a benefit to the treated patient, and it reduces the viral load of treated patients, thus helping to reduce the chance of HIV transmission from the treated patient to the rest of the population.

We performed a simulation study to evaluate the cost-effectiveness of HAART targeted to different population groups in Russia (Long, Brandeau et al. 2006). We considered policies that target treatment only to noninjection drug users, policies that target treatment only to injection drug users, and policies that provide HIV treatment without regard to injection drug using status.

The analysis was based on a dynamic compartmental model of HIV transmission and progression in a population of injection drug users and non-users. Individuals were divided into compartments according to drug using status, HIV status (uninfected, HIV infected and not eligible for treatment, HIV infected and eligible for treatment, and AIDS), and treatment status (receiving HAART or not receiving HAART). We simulated the model over a 20 -year time horizon and, for each treatment policy, we calculated the expected value of net present costs incurred and quality-adjusted life years gained.

The analysis showed that the most cost-effective policy would be to target treatment only to injection drug users. This makes intuitive sense: injection drug users are the key drivers of the epidemic in Russia so reducing their infectivity via HAART could significantly slow the HIV epidemic in Russia. However, such a policy is, not surprisingly, politically infeasible. The next most costeffective policy is to treat patients regardless of injection drug using status. The current policy of treating only non-injection drug users is the least cost-effective policy. The policy conclusion from this work is that HIV treatment resources in Russia should be allocated to all eligible patients.

\subsection{Reducing Concurrent Partnerships in Sub- Saharan Africa}

In sub-Saharan Africa, HIV is spread primarily through heterosexual contact. In addition to risky sexual contacts, many individuals have more than one partner (so-called concurrent partnerships). A number of countries in subSaharan Africa have implemented so-called "ABC campaigns", which focus on Abstinence, Being faithful, and Condom use. However, abstinence programs and condom use programs have not been completely successful. Thus, many experts believe that programs aimed at concurrency reduction (with the message to "Be faithful" to one partner) are the best hope for controlling the spread of HIV in sub-Saharan Africa (Potts, Halperin et al. 2008). Indeed, several countries in sub-Saharan Africa, such as Uganda and Botswana, have instituted nationwide programs aimed at concurrency reduction.

Several researchers have investigated the effects of concurrency on the spread of infectious diseases (e.g., (Watts and May 1992; Kretzschmar and Morris 1996)) but no study has evaluated the cost-effectiveness of programs aimed at reducing concurrency. In order to properly evaluate the effects of concurrency on the spread of HIV, and the effects of programs aimed at reducing such concurrency, a microsimulation of individuals in a social network is needed.

We developed a simulation model of 10,000 individuals in a network (5,000 males and 5,000 females). For each individual, we keep track of age, gender, HIV infection status, HIV treatment status, and number and type of sexual partners. We distinguish two types of sexual partnerships, marital partnerships (which are long term and generally involve unprotected sexual contact) and nonmarital partnerships (which are often in addition to marital partnerships, and may involve a higher rate of protected sexual contact). We simulate the model over a ten-year time horizon using a time increment of one month. In any one month, an individual may dissolve or form a new sexual partnership; may become HIV infected; may progress to a more advanced HIV disease stage; may enter treatment; may die, or may mature out of the population. Additionally, new (uninfected) individuals mature into the population each month. All of these events are stochastic.

We are currently implementing the model with data from South Africa. We will use the model to evaluate two different potential effects of concurrency reduction programs: a reduction in the average number of sexual partnerships (above 1) that an individual has at any point in time, and an increase in the length of existing partnerships. For each potential change in concurrency parameters, we will measure net present cost incurred and quality-adjusted life years experienced over the time horizon of the problem. 


\section{Brandeau}

\section{CONTACT TRACING}

Contact tracing, also known as partnership notification, is a key means of controlling low-prevalence infectious diseases. Health departments in the United States are required by law to trace contacts of people found to be infected with tuberculosis. In addition, contact tracing is often performed for a number of sexually transmitted diseases such as Chlamydia and gonorrhea. Contact tracing is often carried out in conjunction with disease screening: when infected individuals are identified by screening, their contacts are then traced.

How much screening and contact tracing should be performed? Intuitively, we would expect that disease screening and contact tracing will have diminishing returns to scale: as more cases are identified, and disease prevalence decreases, it becomes more costly to find each new case. Moreover, the contacts of these index cases will have a lower likelihood of being infected when disease prevalence is lower.

We developed a simulation model of contact tracing and the spread of an infectious disease in a network of individuals (Armbruster and Brandeau 2007). We considered a disease that is endemic in a population of $n$ individuals. We modeled individuals as nodes in an undirected graph and, for each individual, we considered four possible disease states: susceptible, infected, recovered, and immune. We modeled the relationships between individuals using random small-world graphs (Watts and Strogatz 1998; Watts 1999). We modeled the effects of screening and contact tracing on the identification and treatment of individuals. We simulated the model for five years in one-day time increments and then, for each control policy we considered, we took the average of 1600 runs.

The analysis showed that, as expected, screening and contact tracing have diminishing returns to scale. More importantly, such a model can be used to determine the cost-effective level of screening and contact tracing, given a specified level of willingness to pay (expressed as the amount one is willing to pay to gain one quality-adjusted year of life). As an example, we implemented the model with data to evaluate contact tracing for control of Chlamydia. We showed that complete disease eradication is not cost-effective; instead screening and contact tracing should be implemented at an intensity that achieves the cost-effective (nonzero) level of disease prevalence. The ultimate goal of this work is to provide a tool that public health departments can use to determine the appropriate level of screening and contact tracing for the various infectious diseases that they monitor and control.

\section{HEPATITIS B PREVENTION AND CONTROL}

Hepatitis B is a blood borne infectious disease that afflicts some 350 million people worldwide (Custer, Sullivan et al. 2004). It is spread perinatally (from mother to child), via sexual contact, and via other blood contact (such as cuts or shared toothbrushes). In the United States, prevalence of hepatitis B is approximately $0.5 \%$ (Custer, Sullivan et al. 2004), but among Asian and Pacific Islanders in the United States, prevalence is approximately $10 \%$ (Chao, Lee et al. 2004; Guane, Siu et al. 2004; Centers for Disease Control and Prevention (CDC) 2006). If left untreated, chronic hepatitis B infection leads to death from liver disease in approximately $25 \%$ of patients. A highly effective, inexpensive vaccine against hepatitis $B$ has been available since the mid-1980s.

We used a simulation model, in conjunction with a decision model, to evaluate the cost-effectiveness of various strategies for screening and catch-up vaccination targeted to adult Asian and Pacific Islanders in the United States (Hutton, So et al. 2007). We simulated the progression of hepatitis B in infected patients: once a patient is exposed to hepatitis $\mathrm{B}$, his immune system can resolve the infection, in which case he is immune from further infection, or the infection can become chronic (lifelong). Chronic infection can progress to diminished liver function, to cirrhosis, to liver cancer, and/or to death. Treatment can slow the progression of chronic infection.

We considered a cohort of 10,000 individuals and simulated the health status of each individual over the course of his or her lifetime in time increments of one year. We measured all costs incurred and qualityadjusted life years experienced in the cohort. We evaluated a universal vaccination strategy in which all adults are vaccinated; a screen-and vaccinate strategy in which individuals are first screened to determine whether they are infected, and then individuals found to be uninfected are vaccinated; a screen-and-treat strategy in which individuals are screened and those found to be chronically infected are put on (lifetime) treatment; and a screen-treatand-ring-vaccinate strategy which involves screening for all, treatment for those people found to be infected, and vaccination of the close contacts of those found to be infected (so-called ring vaccination).

The analysis showed that universal vaccination of adult Asian and Pacific Islanders is not cost-effective because their chance of acquiring chronic hepatitis B infection as an adult is low. However, screening followed by treatment, and screening followed by treatment and ring vaccination, are both highly cost-effective. The results of this analysis influenced the revised hepatitis B control guidelines recently issued by the Centers for Disease Control and Prevention (http://www.cdc.gov/mmwr/preview/ mmwrhtml/rr5708a1.htm?s_cid=rr5708a1_x). 


\section{Brandeau}

Recently we completed an analysis of hepatitis B catch-up vaccination for school children in China (Hutton, So et al. 2008). Approximately 120 million people in China have chronic hepatitis B infection (Liu and Fan 2007) and, although the Chinese government has made significant progress in improving newborn hepatitis B vaccination coverage, many children and adolescents still remain unprotected from the virus. Using a simulation model similar to the one we used for the United States study (but adjusted to account for differences in the chance that children acquire chronic infection, as a function of age), we evaluated catch-up vaccination policies targeted to children ages 1-19. The analysis showed that such vaccination is cost-saving.

\section{ACKNOWLEDGMENTS}

Margaret Brandeau is supported by Grant Number R01DA15612 from the National Institute on Drug Abuse.

\section{AUTHOR BIOGRAPHY}

MARGARET L. BRANDEAU is a Professor of Management Science and Engineering and Professor of Medicine (by Courtesy) at Stanford University. She received her BS and MS degrees from MIT and her PhD in Engineering-Economic Systems from Stanford University. Dr. Brandeau has published two books and numerous refereed journal articles. She is a recipient of the INFORMS President's Award, the Pierskalla Prize from INFORMS (for research excellence in health care management science), a Best Paper Award from the Society for Computer Simulation, and a Presidential Young Investigator Award. Her research focuses on health policy modeling, with current projects in HIV prevention and treatment, hepatitis B prevention and control, and bioterror preparedness planning. She is a member of INFORMS.

\section{REFERENCES}

AIDS Foundation East West. 2005. Officially Registered HIV Cases in the Russian Federation: 1 January 1987 through 2 May $2005 . \quad$ Retrieved August 15, 2005, from http://www.afew.org/english/ statistics/HIVdata-RF.htm.

Anderson, R. M. and R. M. May. 1991. Infectious diseases of humans: Dynamics and control. Oxford, Oxford University Press.

Armbruster, B. and M. L. Brandeau. 2007. Contact tracing to control infectious disease: When enough is enough. Health Care Management Science 10: 34155.

Centers for Disease Control and Prevention (CDC). 2006. Screening for chronic hepatitis B among Asian/Pacific Islander populations--New York City,
2005. MMWR Morbidity and Mortality Weekly Report 55(18): 505-9.

Chao, S., P. V. Lee, W. Prapong, et al. 2004. High prevalence of chronic hepatitis B (HBV) infection in adult Chinese Americans living in California [Abstract]. Hepatology 40(Suppl 1): 717A.

Custer, B., S. D. Sullivan, T. K. Hazlet, et al. 2004. Global epidemiology of hepatitis B virus. Journal of Clinical Gastroenterology 38(10 Suppl): S158-68.

Gold, M. R., J. E. Siegel, L. B. Russell, et al. 1996. Costeffectiveness in health and medicine. New York, Oxford University Press.

Guane, R., P. Siu, K. Lam, et al. 2004. Prevalence of HBV and risk for HBV acquisition in hepatitis B screening programs in large metropolitan cities in the United States [Abstract]. Hepatology 40(S4): 716A.

Hutton, D. W., S. K. So and M. L. Brandeau. 2007. Costeffectiveness of screening and vaccinating adult Asian and Pacific adults for hepatitis B. Annals of Internal Medicine 147(7): 460-469.

Hutton, D. W., S. K. So and M. L. Brandeau. 2008. Costeffectiveness of hepatitis B catch-up vaccination among children and adolescents in China. Working paper.

Joint United Nations Programme on HIV/AIDS (UNAIDS) (2004). 2004 report on the global AIDS epidemic. Geneva, Switzerland, United Nations.

Kretzschmar, M. and M. Morris. 1996. Measures of concurrency in networks and the spread of infectious disease. Mathematical Biosciences 133: 165-95.

Liu, J. and D. Fan. 2007. Hepatitis B in China. The Lancet 369(9573): 1582-1583.

Long, E. F., M. L. Brandeau, C. M. Galvin, et al. 2006. Slowing the HIV epidemic in St. Petersburg, Russia: Effectiveness and cost-effectiveness of expanded antiretroviral therapy. AIDS 20: 2207-15.

Potts, M., D. T. Halperin, D. Kirby, et al. 2008. Reassessing HIV prevention. Science 320(5877): 749-750.

Rhodes, T., A. Sarang, A. Bobrik, et al. 2004. HIV transmission and HIV prevention associated with injecting drug use in the Russian Federation. International Journal of Drug Policy 15(1): 1-16.

Watts, C. H. and R. M. May. 1992. The influence of concurrent partnerships on the dynamics of HIV/AIDS. Mathematical Biosciences 108: 89-104.

Watts, D. 1999. Networks, dynamics, and the small-world phenomenon. American Journal of Sociology 105(2): 493-527.

Watts, D. J. and S. H. Strogatz. 1998. Collective dynamics of 'small-world' networks. Nature 393: 440-442.

World Health Organization. 1999. Removing Obstacles to Healthy Development. Retrieved October 3, 2008, from http://www.who.int/infectious_disease_report.

World Health Organization. 2000. Millennium Development Goals. MDG6. Combat HIV/AIDS, Malaria and 


\section{Brandeau}

Other Diseases. Retrieved October 3, 2008, from http://www.who.int/topics/ millennium_ development_goals/diseases/en/.

World Health Organization. 2007. Global HIV prevalence has levelled off. from http://www.who.int/ mediacentre/news/releases/2007/pr61/en/.
World Health Organization (WHO) (2005). Summary country profile for HIV/AIDS treatment scale-up: Russian Federation, June 2005, World Health Organization. 2005. 